\title{
On the uniqueness of stable anisotropic capillary hypersurfaces that contact the edge of a wedge
}

\author{
Motoki Oka ${ }^{1 *}$ \\ ${ }^{1}$ Graduate School of Mathematics, Kyushu University, 744, Motooka, Nishi-ku, Fukuoka 819- \\ 0395, Japan \\ *Corresponding author: oka.motoki.554@s.kyushu-u.ac.jp
}

Received April 25, 2021, Accepted August 31, 2021

\begin{abstract}
Anisotropic energies serve to model the boundaries of some types of crystals and liquid crystals. We study the stability of capillary hypersurfaces with anisotropic energy in a wedge, which contact with the edge of the wedge. We prove that stable capillary hypersurfaces in a wedge are, up to rescaling, only parts of the Wulff shape under some boundary conditions. Here the Wulff shape is the model of a single crystal introduced by G. Wulff.
\end{abstract}

Keywords anisotropic energy, capillary surface, crystal, Wulff shape

Research Activity Group Geometric Shape Generation

\section{Introduction}

We study equilibrium hypersurfaces in the $(n+1)$ dimensional Euclidean space $\mathbb{R}^{n+1}$ for anisotropic surface energies. At first, we introduce anisotropic energies and the Wulff shape. Let $\gamma$ be a positive continuous function on the $n$-dimensional unit sphere $S^{n}$ and let $X$ : $M \rightarrow \mathbb{R}^{n+1}$ be an immersion from an $n$-dimensional oriented connected compact $C^{\infty}$ manifold $M$ with $\partial M \neq \emptyset$ into $\mathbb{R}^{n+1}$. Let $\nu$ be the Gauss map of $X$, that is, $\nu$ is the unit normal vector field along $X$. The anisotropic energy of $X$ is defined as $\mathcal{F}_{\gamma}(X):=\int_{M} \gamma(\nu) d A$, where $d A$ is the $n$-dimensional volume form of $M$ induced by $X$. This energy was introduced by J. W. Gibbs in order to model the shape of small crystals. It is known that, for any positive number $V>0$, among all closed hypersurfaces as above enclosing the same $(n+1)$-dimensional volume $V$, there exists a unique (up to translation in $\mathbb{R}^{n+1}$ ) minimizer $W_{\gamma}(V)$ of $\mathcal{F}_{\gamma}$ [1]. The minimizer $W_{\gamma}=W_{\gamma}\left(V_{0}\right)$ for the specific value $V_{0}:=(n+1)^{-1} \int_{S^{n}} \gamma(\nu) d S^{n}$ is called the Wulff shape for $\gamma$. The Wulff shape is a model of small crystals introduced by G. Wulff [2]. When $\gamma \equiv 1$, $\mathcal{F}_{\gamma}$ is the area of $X$ and $W_{\gamma}$ is the unit sphere. In general, the Wulff shape is not smooth, but if $\gamma$ is of class $C^{2}$ and has a certain convexity (that is, $n \times n$ matrix $D^{2} \gamma+\gamma I_{n}$ is positive definite at each point on $S^{n}$, denoted by $D^{2} \gamma+\gamma I_{n}>0$, where $D^{2} \gamma$ denotes the Hessian of $\gamma$ on $S^{n}$ and $I_{n}$ is the identity map on $T_{x} S^{n}$ $\left.\left(\forall x \in S^{n}\right)\right)$, then $W_{\gamma}$ is a smooth strictly convex closed hypersurface.

Recently, variational problems for hypersurfaces with anisotropic energy have been studied by many authors. Concerning the uniqueness of stable solutions, B. Palmer showed that stable, connected, oriented, closed equilibrium hypersurfaces are (up to translations and homothety) the Wulff shape [3] (stable closed hypersurface is defined similarly to the definition of stable anisotropic hypersurface which will be given in Definition 3). In this

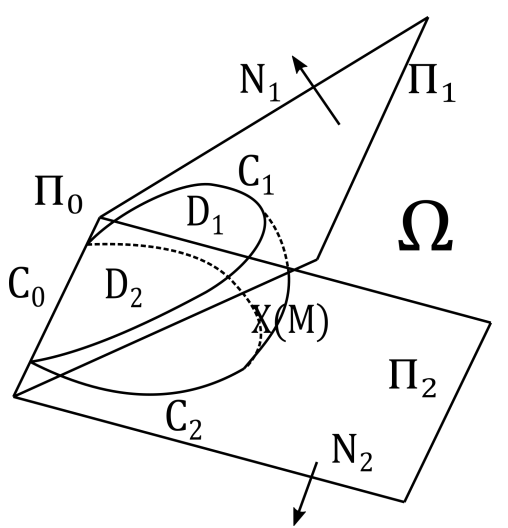

Fig. 1. A surface $X(M)$ in a wedge type domain $\Omega$

paper, we study a free boundary problem for hypersurfaces with anisotropic energy whose supporting surfaces form a wedge shape. The hypersurfaces we study are as follows (Fig 1). Let $\Pi_{1}, \Pi_{2} \subset \mathbb{R}^{n+1}$ be $n$-dimensional closed half hyperplanes which are not parallel to each other and $\partial \Pi_{1}=\partial \Pi_{2}$, and we consider the wedge-like domain $\Omega \subset \mathbb{R}^{n+1}$ whose boundary is $\Pi_{1} \cup \Pi_{2}$. Let $N_{i}$ be the unit normal vector field on $\Pi_{i}$ outward from $\Omega$ $(i=1,2)$. Note that the pair $N_{1}, N_{2}$ is linearly independent. We call $\Pi_{0}:=\Pi_{1} \cap \Pi_{2}$ the edge of the wedge. Let $X: M \rightarrow \mathbb{R}^{n+1}$ be an immersion from an $n$-dimensional oriented connected compact $C^{\infty}$ manifold $M$ with $\partial M \neq$ $\emptyset$ into $\bar{\Omega}$ whose supporting surface is $\Pi_{1} \cup \Pi_{2}$ and satisfying $\partial X(M) \cap \Pi_{i} \neq \emptyset(i=1,2)$. We assume that $\partial M$ is homeomorphic to $S^{n-1}$ and $X(M) \cap \Pi_{0} \neq \emptyset$. Furthermore, we assume that the restriction of $X$ to $\partial M$ is an embedding. Let $C_{i}:=\partial X(M) \cap \Pi_{i}(i=1,2)$ and $\sigma_{i}:=X^{-1}\left(C_{i}\right)(i=1,2)$. And set $\sigma_{0}:=\sigma_{1} \cap \sigma_{2}$ and $C_{0} \subset \Pi_{0}$ to be the $(n-1)$-dimensional domain bounded by $X\left(\sigma_{0}\right)$. Let $D_{i} \subset \Pi_{i}$ be the $n$-dimensional domain bounded by $C_{i}$ and $C_{0}(i=1,2)$. We denote by $V(X)$ the volume bounded by $X(M)$ and $\Pi_{1} \cup \Pi_{2}$. For these 
kinds of hypersurfaces, we define the total energy as follows.

Definition 1 (Energy). Let $\omega_{1}, \omega_{2}$ be real constants. We define the wetting energy $\mathcal{W}(X)$ of $X$ as

$$
\mathcal{W}(X):=\omega_{1} \mathcal{H}^{n}\left(D_{1}\right)+\omega_{2} \mathcal{H}^{n}\left(D_{2}\right),
$$

where $\mathcal{H}^{n}$ is the $n$-dimensional Hausdorff measure. We define the total energy $E(X)$ of $X$ as

$$
E(X):=\mathcal{F}_{\gamma}(X)+\mathcal{W}(X),
$$

where $\gamma$ is of class $C^{2}$ and $D^{2} \gamma+\gamma I_{n}>0$. We call $\omega_{i}$ the wetting constants.

Let $\tilde{X}:(-\varepsilon, \varepsilon) \times M \rightarrow \mathbb{R}^{n+1}$ be a smooth variation of $X(\varepsilon>0)$. If $\tilde{X}$ satisfies the following conditions, then $\tilde{X}$ is said to be admissible:

- $\tilde{X}(t, x) \in \Pi_{1} \cup \Pi_{2}(\forall t \in(-\varepsilon, \varepsilon), \forall x \in \partial M)$,

- $V\left(X_{t}\right)=V(X)(\forall t \in(-\varepsilon, \varepsilon))$,

where $X_{t}=\tilde{X}(t, \cdot)$.

Definition 2. Let $X: M \rightarrow \bar{\Omega}$ be a hypersurface as above. $X$ is called an anisotropic capillary hypersurface if

$$
\delta E:=\left.\frac{d E\left(X_{t}\right)}{d t}\right|_{t=0}=0
$$

for any admissible variation $\tilde{X}$ of $X$.

We define the stability of anisotropic capillary hypersurfaces as follows.

Definition 3. Let $X: M \rightarrow \bar{\Omega}$ be an anisotropic capillary hypersurface as above. $X$ is said to be stable if

$$
\left.\frac{d^{2} E\left(X_{t}\right)}{d t^{2}}\right|_{t=0} \geq 0
$$

for any admissible variation $\tilde{X}$ of $X$.

M. Koiso proved that stable anisotropic capillary hypersurfaces that do not contact the edge of a wedge are (up to translations and homothety) a part of the Wulff shape [4]. We prove the following uniqueness theorem for stable anisotropic capillary hypersurfaces in the wedge, which do contact the edge as follows.

Theorem 4. Assume that $\gamma: S^{n} \rightarrow \mathbb{R}_{>0}$ is of class $C^{2}$ and that $D^{2} \gamma+\gamma I_{n}>0$ holds, and let $X: M \rightarrow \bar{\Omega}$ be an anisotropic capillary hypersurface. We assume that $0 \leq \omega_{i}<\gamma\left(N_{i}\right)(i=1,2)$. If $X$ is stable and in addition, if we assume that $D_{1}$ and $D_{2}$ are convex when $n \geq 3$, then $X(M)$ is (up to translation and homothety) a part of the Wulff shape $W_{\gamma}$. Conversely, if $X$ is an embedding onto a part of $W_{\gamma}$ (up to translation and homothety), then it is stable.

Remark 5. Y. Mitsuo proved Theorem 4 in the case $\gamma \equiv 1$ [5]. So Theorem 4 is a generalization of [5].

Some parts of long computations are omitted in this paper. They will appear in the forthcoming paper by the author.

\section{Preliminaries}

In this section, we give some of the fundamental definitions and formulas which will be needed in the proof of

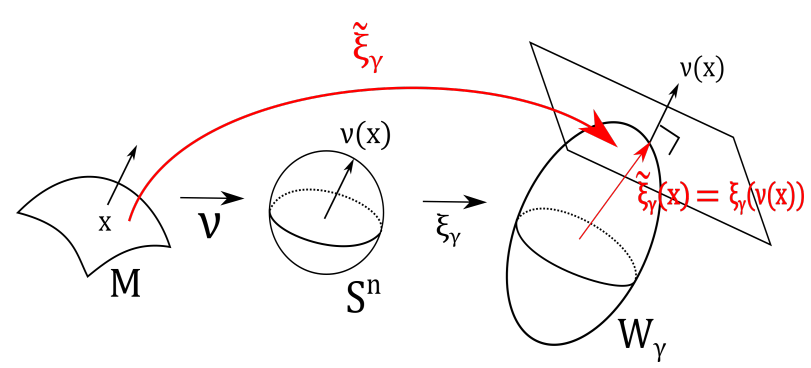

Fig. 2. Cahn-Hoffman field

Theorem 4. For details, see $[3,4,6]$. Let $X: M \rightarrow \mathbb{R}^{n+1}$ be an immersion from an oriented connected compact differentiable manifold $M$ with or without boundary into $\mathbb{R}^{n+1}$. Let $\gamma$ be a positive continuous function on the unit sphere $S^{n} \subset \mathbb{R}^{n+1}$. In general, the Wulff shape $W_{\gamma}$ for $\gamma$ is not smooth (it is, however, a convex closed surface), but if $\gamma$ is of class $C^{2}$ and $D^{2} \gamma+\gamma I_{n}>0$, then $W_{\gamma}$ is smooth and strictly convex. So, in the following, we assume that $\gamma: S^{n} \rightarrow \mathbb{R}_{>0}$ is of class $C^{2}$ and $D^{2} \gamma+\gamma I_{n}>0$. We define the Cahn-Hoffman map $\xi_{\gamma}$ of $\gamma$ as follows (Fig. 2):

$$
\xi_{\gamma}: S^{n} \rightarrow \mathbb{R}^{n+1}, \quad \xi_{\gamma}(\nu):=D \gamma+\gamma(\nu) \nu\left(\nu \in S^{n}\right),
$$

where $D$ is the gradient on $S^{n}$. It is known that if $D^{2} \gamma+$ $\gamma I_{n}>0$, then $W_{\gamma}$ is the image of $\xi_{\gamma}\left(\xi_{\gamma}\left(S^{n}\right)=W_{\gamma}\right)$. The composite map $\tilde{\xi}_{\gamma}:=\xi_{\gamma} \circ \nu: M \rightarrow \mathbb{R}^{n+1}$ is called the Cahn-Hoffman field (or the anisotropic Gauss map) of $X$ where $\nu: M \rightarrow S^{n}$ is the Gauss map of $X$. If $\gamma \equiv 1$, then $\tilde{\xi}_{\gamma}$ is the usual Gauss map of $X$. Hence, the CahnHoffman field is a generalization of the Gauss map of $X$. Now we define the anisotropic shape operator $S^{\gamma}$ of $X$ as follows:

$$
S_{p}^{\gamma}:=-d \tilde{\xi}_{\gamma}: T_{p} M \rightarrow T_{\tilde{\xi}(p)} \tilde{\xi}_{\gamma}(M) \simeq T_{p} M .
$$

By using the fact that $\xi_{\gamma}$ is the inverse of the Gauss map of $W_{\gamma}$, the last isomorphism is proved. Using the anisotropic shape operator, we define the anisotropic curvatures of $X$ :

Definition 6 (Anisotropic curvatures [6]). (i) The eigenvalues of $S^{\gamma}$ are called the anisotropic principal curvatures of $X$, denoted by $k_{1}^{\gamma}, \ldots, k_{n}^{\gamma}$.

(ii) Let $\sigma_{r}^{\gamma}$ be the elementary symmetric functions of $k_{1}^{\gamma}, \ldots, k_{n}^{\gamma}$ :

$$
\sigma_{r}^{\gamma}:=\sum_{1 \leq l_{1}<\cdots<l_{r} \leq n} k_{l_{1}}^{\gamma} \ldots k_{l_{r}}^{\gamma}
$$

$(r=1, \ldots, n)$, where $\sigma_{0}^{\gamma}:=1 . H_{r}^{\gamma}:=\sigma_{r}^{\gamma} /{ }_{n} C_{r}$ is called the $r$ th anisotropic mean curvature of $X$, where ${ }_{n} C_{r}=n ! /(k !(n-k) !)$.

(iii) We define the anisotropic mean curvatures $\Lambda$ as $\Lambda:=H_{1}^{\gamma}=1 / n \sum_{i=1}^{n} k_{i}^{\gamma}$.

Remark 7. (i) If $d \xi_{\gamma}=D^{2} \gamma+\gamma I_{n}$ is positive definite at a point $\nu(p)(p \in M)$, then all of the anisotropic principal curvatures of $X$ at $p$ are real. 


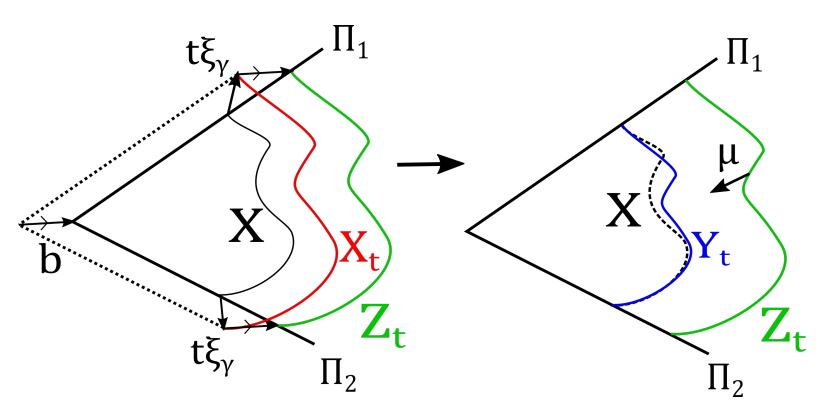

Fig. 3. Construction of the specific admissible variation $Y_{t}$

(ii) The $k_{i}^{\gamma}$ are not real in general. However, $H_{r}^{\gamma}$ are always real.

(iii) The anisotropic mean curvatures satisfy $\Lambda=$ $1 / n\left(-\operatorname{div}_{M} D \gamma+n H\right)$ where $H$ is the mean curvature of $X$.

Next, we introduce a useful formula, which is called the Steiner-type formula. This is a generalization of Weyl's tube formula [7].

Fact 8 (Steiner-type formula cf. [6]). Assume that $\gamma$ : $S^{n} \rightarrow \mathbb{R}_{>0}$ is of class $C^{2}$. Let $X: M \rightarrow \mathbb{R}^{n+1}$ be an immersion from an $n$-dimensional oriented connected compact manifold $M$ with or without boundary into $\mathbb{R}^{n+1}$. We define the anisotropic parallel hypersurface $X_{t}$ as

$$
X_{t}:=X+t \xi_{\gamma}: M \rightarrow \mathbb{R}^{n+1}(t \in \mathbb{R}) .
$$

Then, the anisotropic energy $\mathcal{F}_{\gamma}\left(X_{t}\right)$ of $X_{t}$ has the following representation:

$$
\mathcal{F}_{\gamma}\left(X_{t}\right)=\int_{M} \gamma(\nu) \sum_{r=0}^{n}(-1)^{r} t^{r}\left({ }_{n} C_{r}\right) H_{r}^{\gamma} d A .
$$

\section{Proof of Theorem 4}

\subsection{Euler-Lagrange equations}

Let $X: M \rightarrow \bar{\Omega}$ be an anisotropic capillary hypersurface in the wedge-like closed domain $\bar{\Omega}$ which does contact with the edge of the wedge defined in the introduction. First, we can prove that the Euler-Lagrange equations of the variational problem for the energy $E$ with free boundary and volume constraint by the same way as the proof of Lemma 1 in [4].

Proposition 9 (Euler-Lagrange equations cf. [4]). $X$ is an anisotropic capillary hypersurface if and only if the following conditions (i) and (ii) hold:

(i) The anisotropic mean curvature $\Lambda$ of $X$ is constant on $M$.

(ii) $\left\langle\tilde{\xi}, N_{i}\right\rangle=\omega_{i}$ on $C_{i}(i=1,2)$.

These conditions imply that an anisotropic capillary hypersurface is a generalization of the classical capillary hypersurface in the absence of gravity.

3.2 An admissible variation constructed by using parallel variation

In order to prove Theorem 4, as [4], we will compute the second variation of the total energy for a specific ad- missible variation as follows (Fig 3). First, we consider the anisotropic parallel hypersurface $X_{t}=X+t \tilde{\xi}(t \in \mathbb{R}$, $|t| \ll 1)$. If $\omega_{1}, \omega_{2}>0$, then $X_{t}$ does not necessarily satisfy the boundary condition. But, since the condition (ii) in Proposition 9 and the pair $N_{1}, N_{2}$ is linearly independent, we can take a suitable translation $Z_{t}:=X_{t}+t b$ $\left(\exists b \in \mathbb{R}^{n+1}\right.$ ) so that $Z_{t}$ satisfies the boundary condition. But $Z_{t}$ is not in general, volume preserving. Hence we take a suitable homothety

$$
Y_{t}:=\mu(t) Z_{t}=\mu(t)(X+t(\tilde{\xi}+b)),
$$

where $\mu(t)$ satisfies $\mu(t)>0, \mu(0)=1$ and $V\left(Y_{t}\right)=$ $V\left(Y_{0}\right)(\forall t)$. Therefore $Y_{t}$ is an admissible variation of $X$. We will compute the second variation of the total energy for the variation $Y_{t}$.

\subsection{Anisotropic energies for the boundary of a hyper- surface}

In order to compute the second variation, we will define a new anisotropic energy for the boundary of considered hypersurfaces. First, we define the anisotropic energy for $C_{i}$. Since $\omega_{i}<\gamma\left(N_{i}\right)(i=1,2)$, we can define the $n$-dimensional cross section domain of $W_{\gamma}$ by the plane $P_{i}$ defined as follows

$$
P_{i}:=\left\{x \in \mathbb{R}^{n+1} \mid\left\langle x, N_{i}\right\rangle=\omega_{i}\right\}(i=1,2) .
$$

Then, set $\hat{o}_{i}=\omega_{i} N_{i}$. It is clear that $P_{i}$ is parallel to $\Pi_{i}$. We denote by $\hat{W}_{i}$ the boundary of the cross section domain (the left side of Fig. 4). We can regard $\hat{o}_{i}$ as the origin of $P_{i}$. Since $\hat{W}_{i}$ is convex and since $\hat{o}_{i}$ is in the inside of $\hat{W}_{i}$, there is a positive support function $\hat{\gamma}_{i}: S^{n-1} \rightarrow \mathbb{R}_{>0}$ such that its Wulff shape is $\hat{W}_{i}$. For later use, let $\xi_{i}$ be the Cahn-Hoffman map for $\hat{\gamma}_{i}$. Now, we define the anisotropic energy of $\chi_{i}:=\left.X\right|_{\sigma_{i}}$ by

$$
\hat{\mathcal{F}}\left(\chi_{i}\right):=\int_{\sigma_{i}} \hat{\gamma}_{i}(\rho) d s
$$

where $d s$ is the volume element of $\chi_{i}$ and $\rho$ is the outward unit normal of $\sigma_{i}$ in the plane $\Pi_{i}$. By using this anisotropic energy, we can show the balancing formula (13) below. Set $\hat{\mathcal{F}}_{i}\left(D_{i}\right):=\hat{\mathcal{F}}_{i}\left(\chi_{i}\right)+h_{i} \mathcal{H}^{n-1}\left(C_{0}\right)$, where let $h_{i}:=\left\langle\tilde{\xi}_{i}(x), \rho_{0}\right\rangle,\left(x \in \sigma_{0}\right)$ where $\rho_{0}$ is the unit outward normal vector on $C_{0}$ from $D_{i}$. Then, we can prove that $h_{i}$ is constant in $x$.

\section{Lemma 10.}

$$
\hat{\mathcal{F}}_{i}\left(D_{i}\right)=-n \Lambda \mathcal{H}^{n}\left(D_{i}\right)(i=1,2) .
$$

Secondly, we define an anisotropic energy for $C_{0}$. Since $\tilde{\xi}\left(\sigma_{0}\right) \subset P_{1} \cap P_{2}$, we can consider the convex hull of $\tilde{\xi}\left(\sigma_{0}\right)$ in $P_{1} \cap P_{2}$, denoted by $W_{0}$ (the right side of Fig. 4 ). Since $W_{0}$ is convex, there is a support function $\hat{\gamma}_{0}: S^{n-2} \rightarrow$ $\mathbb{R}_{>0}$ such that its Wulff shape is $\hat{W}_{0}:=\partial W_{0}$. Let $\xi_{0}$ be the Cahn-Hoffman map of $\hat{\gamma}_{0}$. We define the anisotropic energy of $\chi_{0}:=\left.X\right|_{\sigma_{0}}$ by

$$
\hat{\mathcal{F}}_{0}\left(\chi_{0}\right):=\int_{\sigma_{0}} \hat{\gamma}_{0}(\eta) d \tilde{s}
$$

where $\eta$ is the outward normal vector field along $\partial C_{0}$ and $d \tilde{s}$ is the volume element of $\partial C_{0}$.

Remark 11. If $n=2$, then $\hat{\mathcal{F}}_{0}\left(\chi_{0}\right)=\mathcal{H}^{1}\left(W_{0}\right)$ is satis- 

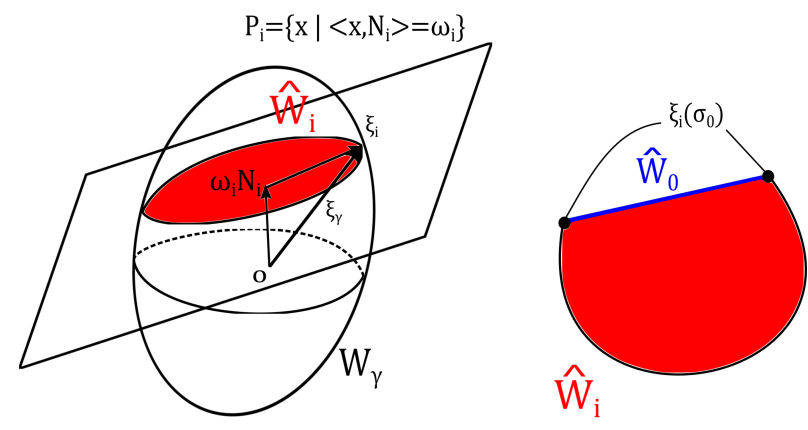

Fig. 4. $\hat{W}_{i}$ (left, the green domain) and $W_{0}$ (right, the blue domain (line))

fied.

\subsection{Second variation of $E\left(Y_{t}\right)$}

First of all, the following proposition can be proved.

\section{Lemma 12.}

$$
\frac{d}{d t} V\left(X_{t}\right)=E\left(X_{t}\right)=\mathcal{F}_{\gamma}\left(X_{t}\right)+\sum_{i=1}^{2} \omega_{i} \mathcal{H}^{n}\left(D_{i}^{t}\right),
$$

where $D_{i}^{t}$ is the $n$-dimensional domain in $\Pi_{i}+t \tilde{\xi}$ bounded by $C_{i}+t \tilde{\xi}$ and the $(n-1)$-dimensional domain enclosed by $X_{t}\left(\sigma_{0}\right)(i=1,2)$.

We can rewrite the two terms of the right hand side of (15) as a power series in $t$ explicitly. By using the Steiner-type formula (9), we have

$$
\begin{aligned}
\mathcal{F}_{\gamma}\left(X_{t}\right)=\mathcal{F}_{\gamma}(X)-n \Lambda \mathcal{F}_{\gamma}(X) t \\
\quad+{ }_{n} C_{2} \int_{M} \gamma(\nu) H_{2}^{\gamma} d A \cdot t^{2}+O\left(t^{3}\right) .
\end{aligned}
$$

Next, we will compute $\mathcal{H}^{n}\left(D_{i}^{t}\right)$. By using the anisotropic energy (12) and (14) of $C_{i}$ and $C_{0}$, respectively, we can prove,

$$
\begin{aligned}
& \mathcal{H}^{n}\left(D_{i}^{t}\right) \\
& =\mathcal{H}^{n}\left(D_{i}\right)+\hat{\mathcal{F}}_{i}\left(D_{i}\right) t \\
& \quad-\frac{n-1}{2}\left(\int_{\sigma_{i}} \hat{\gamma}_{i}(\rho) \hat{\Lambda} d s-\frac{1}{n-1} \hat{\mathcal{F}}_{0}\left(\chi_{0}\right) h_{i}\right) t^{2} \\
& \quad+O\left(t^{3}\right)
\end{aligned}
$$

where $\hat{\Lambda}$ is the anisotropic mean curvature of $C_{i}$.

Substituting (16) and (17) into (15), the total energy $E\left(X_{t}\right)$ can be represented as a power series in $t$. By long computation, we can get the second variation of $E\left(Y_{t}\right)$ as follows:

\section{Lemma 13.}

$$
\begin{aligned}
\left.\frac{d^{2}}{d t^{2}} E\left(Y_{t}\right)\right|_{t=0}=- & (n-1) \sum_{i=1}^{2} \omega_{i} B_{i} \\
& -\frac{1}{n} \int_{M} \gamma(\nu) \sum_{1 \leq i<j \leq n}\left(k_{i}^{\gamma}-k_{j}^{\gamma}\right)^{2} d A
\end{aligned}
$$

where

$$
B_{i}:=\int_{\sigma_{i}} \hat{\gamma}_{i} \hat{\Lambda} d s+\frac{\left(\mathcal{F}_{i}\left(D_{i}\right)\right)^{2}}{n \mathcal{H}^{n}\left(D_{i}\right)}-\frac{1}{n-1} \hat{\mathcal{F}}_{0}\left(\chi_{0}\right) h_{i} .
$$

\subsection{Stability}

Firstly, we can get the following inequalities.

Lemma 14. (1) If $n=2$, then $B_{i} \geq 0$.

(2) If $n \geq 3$ and $D_{i}$ is convex, then $B_{i} \geq 0$.

We must assume $D_{i}$ are convex for $n \geq 3$ in order to prove $B_{i} \geq 0$ since we use the Alexandrov-Fenchel inequality ( $[8$, Theorem 6.3 .1$]$ ), which is a result in convex analysis. If $n \geq 3$, then we assume that $D_{i}$ are convex. If $X$ is stable, then the second variation of the total energy of $X$ for any admissible variations is non-negative. But by Lemmas 13 and 14, we have $\left.\frac{d^{2}}{d t^{2}} E\left(Y_{t}\right)\right|_{t=0} \leq 0$. Therefore, $\left.\frac{d^{2}}{d t^{2}} E\left(Y_{t}\right)\right|_{t=0}=0$ and hence $k_{1}^{\gamma}=\cdots=k_{n}^{\gamma}$ is satisfied. It is known that any hypersurface that satisfies $k_{1}^{\gamma}=\cdots=k_{n}^{\gamma}$ is (up to translation and homothety) a part of the Wulff shape [9]. Conversely, if $X(M)$ is a part of a homothety of the Wulff shape $W_{\gamma}$, then we can prove that $X$ is stable by similar way to the proof of [10, Theorem 4.1]. Therefore, we have proved Theorem 4.

\section{Acknowledgments}

I would like to thank Professor M. Koiso for continuous guidance and support.

\section{References}

[1] J. E. Taylor, Crystalline variational problems, Bull. Amer. Math. Soc., 84 (1978), 568-588.

[2] G. Wulff, Zur Frage der Geschwindigkeit des Wachsthums und der Auflösung der Krystallflächen, Zeitschrift für Krystallographie und Mineralogie, 34 (1901), 449-530.

[3] B. Palmer, Stability of the Wulff shape, Proc. Amer. Math. Soc., 126 (1998), 3661-3667.

[4] M. Koiso, Uniqueness of closed equilibrium hypersurfaces for anisotropic surface energy and application to a capillary problem, Math. Comput. Appl., 24 (2019), 88-103.

[5] Y. Mitsuo, Stable capillary hypersurfaces in a wedge (in Japanese), Master Thesis, Faculty of Mathematics, Kyushu University, (2015).

[6] M. Koiso, Uniqueness of stable closed non-smooth hypersurfaces with constant anisotropic mean curvature, arXiv:1903.03951 [math.DG].

[7] H. Weyl, On the volume of tube, Amer. J. Math., 61 (1939), 461-472.

[8] R. Schneider, Convex Bodies: the Brunn-Minkowski Theory, 2nd edn., Encycl. Math. Appl., 151 Cambridge University Press, Cambridge, 2014.

[9] R. C. Reilly, The relative differential geometry of nonparametric hypersurfaces, Duke Math. J., 43 (1976), 705-721.

[10] M. Koiso and B. Palmer, Anisotropic capillary surfaces with wetting energy, Calc. Var. Partial Differ. Equ., 29 (2007), 295345 . 